\title{
Combining Morphological Information in a Manifold Learning Framework: Application to Neonatal MRI
}

\author{
P. Aljabar ${ }^{1, \star}$, R. Wolz ${ }^{1}$, L. Srinivasan ${ }^{2}$, S. Counsell ${ }^{3}$, J.P. Boardman ${ }^{3}$,
} M. Murgasova ${ }^{1}$, V. Doria ${ }^{2}$, M.A. Rutherford ${ }^{3}$, A.D. Edwards ${ }^{2}$, J.V. Hajnal ${ }^{3}$, and D. Rueckert ${ }^{1}$

${ }^{1}$ Department of Computing, Imperial College London, UK Paul.Aljabar@Imperial.ac.uk

${ }^{2}$ Division of Neonatology, Imperial College Healthcare NHS Trust, London, UK

${ }^{3}$ Institute of Clinical Sciences, Imperial College London and MRC Clinical Sciences Centre, Hammersmith Hospital, London, UK

\begin{abstract}
MR image data can provide many features or measures although any single measure is unlikely to comprehensively characterize the underlying morphology. We present a framework in which multiple measures are used in manifold learning steps to generate coordinate embeddings which are then combined to give an improved single representation of the population. An application to neonatal brain MRI data shows that the use of shape and appearance measures in particular leads to biologically plausible and consistent representations correlating well with clinical data. Orthogonality among the correlations suggests the embedding components relate to comparatively independent morphological features. The rapid changes that occur in brain shape and in MR image appearance during neonatal brain development justify the use of shape measures (obtained from a deformation metric) and appearance measures (obtained from image similarity). The benefit of combining separate embeddings is demonstrated by improved correlations with clinical data and we illustrate the potential of the proposed framework in characterizing trajectories of brain development.
\end{abstract}

\section{Introduction}

A number of machine learning techniques have been developed for converting data from a high to a low dimensional representation more suitable for further processing steps such as clustering or regression. This class of methods, described under the terms 'manifold learning' or 'dimensionality reduction', have recently begun to be applied to the field of medical image analysis. Medical images, or derived features, are natural candidates as raw data for manifold learning where a typical structural magnetic resonance (MR) image, for example, can contain around a million voxels.

The intuition underlying the application of manifold learning to medical images is that, while each image may be viewed as a single point in a very highdimensional space, a set of such points for a population of images may be well

\footnotetext{
^ Corresponding author.
}

T. Jiang et al. (Eds.): MICCAI 2010, Part III, LNCS 6363, pp. 1-8, 2010.
(c) Springer-Verlag Berlin Heidelberg 2010 
represented by sub-manifold of the space that is likely to be non-linear and of a significantly lower dimension. Although it may be possible to learn a manifold directly from the image data, it is more typical to use measures that relate pairs of images in a dataset.

As an example, pairwise similarity (based on model description length) was used in [1] in an application of Laplacian eigenmaps [2] to identify coherent sets of landmarks in images that can be clustered. The 'shape maps' produced have clear applications in medical imaging data where landmark based analysis is common. The Laplacian eigenmap approach was also applied in [3] where similarities between images were derived from structural segmentation overlaps and the resulting embedding was used to discriminate clinical groups of elderly patients with Alzheimer's Disease (AD) and controls. Another manifold learning algorithm, Isomap [4, was used to estimate the manifold structure of brain MRI acquired from $\mathrm{AD}$ patients and controls [5]. In that study, distance measures between image pairs are derived from the non-rigid deformations aligning them. Pairwise distances derived from the deformations were also used to empirically construct a manifold for images in [6] using a k-NN graph building approach. By 'navigating' the resulting sparse graph, in which edges join pairs of similar images represented by the nodes, it becomes possible to estimate transformations between pairs of very different images by concatenating successive transformations between pairs of similar images. Navigation of a low-dimensional coordinate embedding was also used in [7] where it was shown that accurate atlas-based segmentations may be obtained by propagating manually labelled structures from a small group of healthy brain images to a large set of images acquired from subjects with a range of pathology.

In this work, we contribute a framework in which separate manifold learning steps, based on different pairwise measures, can be applied to MR images. The resulting coordinate embeddings are then fused to produce a single combined embedding. The use of multiple pairwise measures allows the decomposition of morphology into different aspects. We give a motivating example using neonatal brain MR images which show both shape changes associated with growth and complementary signal changes due to microscopic structural processes such as myelination. The two pairwise measures used were therefore chosen to represent shape and appearance in the data. We compare the embeddings with nonimaging data for the subjects and the separate shape- and appearance-based embeddings correlate in a consistent way with these clinical data. The results also indicate the benefit of fusing embeddings as this leads to improved correlation and provides a useful representation for characterizing trajectories of change during the important period of neonatal brain development.

\section{Methods}

Our approach begins with registrations to obtain measures reflecting shape and appearance relations for image pairs in the data set. Each measure produces a coordinate embedding via a manifold learning technique appropriate for the measure. The separate embeddings are then fused by scaling and concatenation 
and, after a final dimensionality reduction step, a combined embedding characterizes the manifold in which the image data reside. A schematic illustration of the proposed approach (as applied to neonatal MRI) is provided in Figure 1.

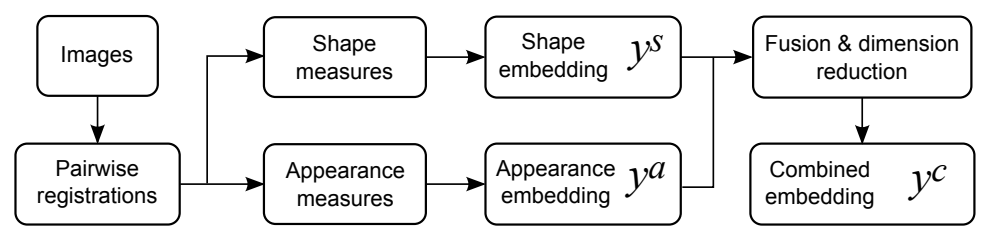

Fig. 1. An overview of the proposed framework as it is applied to neonatal brain MRI

\subsection{Pairwise Measures between Images}

Given images $\mathcal{I}_{A}, \mathcal{I}_{B}: \Omega \subset \mathbb{R}^{3} \rightarrow \mathbb{R}$ and a non-rigid deformation $\phi: \mathbb{R}^{3} \rightarrow \mathbb{R}^{3}$ between them, we derive an appearance measure for the pair from their intensitybased similarity and a shape measure from $\phi$. These measures are chosen for application to neonatal data but other measures possible in different applications.

The intensity similarity of the images is given by a function on the pairings $\left\{\left(\mathcal{I}_{A}(x), \mathcal{I}_{B}(\phi(x))\right): x \in \Omega\right\}$ and we define the pairwise appearance measure to be the normalised cross correlation

$$
\mathcal{M}^{a}\left(\mathcal{I}_{A}, \mathcal{I}_{B} ; \phi\right)=\frac{\sum\left(\mathcal{I}_{A}(x)-\mu_{A}\right)\left(\mathcal{I}_{B}(\phi(x))-\mu_{B}\right)}{\sqrt{\sum\left(\mathcal{I}_{A}(x)-\mu_{A}\right)^{2} \sum\left(\mathcal{I}_{B}(\phi(x))-\mu_{B}\right)^{2}}}
$$

where $\mu_{A}$ and $\mu_{B}$ are the average intensities.

The pairwise shape measure is based on a metric induced by $\phi$. In a diffeomorphic setting, such as the one given in $[8, \phi(x)$ is defined as the point at $t=1$ of an integral curve $\phi(x, t)$ of a time-varying velocity field $v(x, t): \mathbb{R}^{3} \times \mathbb{R} \rightarrow \mathbb{R}^{3}$. The metric induced on an image pair is then defined as the minimal value of the energy integral $\int_{0}^{1}\|v(x, t)\|_{L}^{2} d t$ such that $\phi$ aligns the images and $L$ denotes a choice of differential operator.

Local structure is important when learning the low-dimensional manifold and distances between images in local neighbourhoods play a greater role in characterizing manifold geometry than longer range distances. In our context, this means that transformations between similar images carry more weight. In this case, transformations can be approximated in a small deformation setting: $\phi(x)=x+u(x)$ for a displacement field $u$. We follow the work of 5 and 6 ] in adopting this approximation and define a pairwise shape measure between images as

$$
\mathcal{M}^{s}\left(\mathcal{I}_{A}, \mathcal{I}_{B} ; \phi\right)=\|u(x)\|_{L}^{2}
$$

where $u(x)$ is obtained from registering $\mathcal{I}_{A}$ and $\mathcal{I}_{B}$ and $L=I d+\alpha \nabla$, with $I d$ and $\nabla$ denoting the identity matrix and divergence operator and $\alpha$ is a weighting parameter.

Both the shape and appearance measures depend on the resolution of the transformation that aligns the image pair, i.e. the scale at which local structure 
in the images is aligned. A coarse transformation only accounts for large-scale shape differences between the images; small-scale variations remain to influence the appearance measure, $\mathcal{M}^{a}$, for the images. We found that differences in appearance are more diffuse under a fine transformation and the way image pairs relate is encoded more in the deformation $u(x)$ between them. In this case, the shape measure becomes more informative. These considerations are similar to those arising in voxel-based morphometry (VBM) studies 9] which seek local group differences in aligned image sets. The resolution of the aligning transformations must allow such differences to remain detectable. In contrast, when images are finely aligned, group differences become encoded in the aligning transformations and deformation-based morphometry (DBM) becomes possible [10].

\subsection{Manifold Learning and Fusion of Embeddings}

The pairwise measures for shape $\left(\mathcal{M}^{s}\right)$ and appearance $\left(\mathcal{M}^{a}\right)$ are used as input data for separate manifold learning steps, with each giving a coordinate embedding for the image data. Since $\mathcal{M}^{a}$ represents a similarity measure and $\mathcal{M}^{s}$ a distance measure, manifold learning techniques specific to each type of measure are applied: A Laplacian eigenmap [2] is used for $\mathcal{M}^{a}$ and Isomap [4] is used for $\mathcal{M}^{s}$. In each case, the image data can be viewed as nodes in a graph where edges are assigned weights derived from $\mathcal{M}^{a}$ or $\mathcal{M}^{s}$.

For $N$ images $\mathcal{I}_{1}, \ldots, \mathcal{I}_{N}$, the Laplacian eigenmap approach assigns a weight $W_{i j}$ to each edge measuring the similarity of images $\mathcal{I}_{i}$ and $\mathcal{I}_{j}$. We set $W_{i j}=$ $\mathcal{M}^{a}\left(\mathcal{I}_{i}, \mathcal{I}_{j}\right)$. The method then seeks a coordinate embedding $y_{i}, i=1, \ldots N$ for the data that minimises the cost function:

$$
\sum_{i, j} W_{i j}\left\|y_{i}-y_{j}\right\|^{2}
$$

where $\|\cdot\|$ is the $L_{2}$ norm. The local structure of the data can be modelled by selecting a neighbourhood size $K$ such that $W_{i j}$ is set to zero when $\mathcal{I}_{j}$ is outside the $K$-nearest neighbourhood of $\mathcal{I}_{i}$.

The Isomap algorithm assigns a distance $\mathcal{D}_{i j}$ to edges reflecting the distance between end nodes. We set $\mathcal{D}_{i j}=\mathcal{M}^{s}\left(\mathcal{I}_{i}, \mathcal{I}_{j}\right)$. Local structure is modelled by constructing a sparse graph in which edges are restricted to be those of the $K$ nearest neighbourhood for each node. The manifold structure is then obtained using geodesic distances $D_{i j}$ between all pairs of nodes estimated by summing the values of $\mathcal{D}_{\alpha \beta}$ along the shortest path between each node pair in the graph. A coordinate embedding $y_{i}, i=1, \ldots N$ is then generated to minimise the cost function

$$
\sum_{i, j}\left(D_{i j}-\left\|y_{i}-y_{j}\right\|\right)^{2}
$$

Isomap and Laplacian eigenmaps are examples of spectral methods since they produce embedding coordinates from eigendecompositions of matrices derived from the edge weights of the graph. Although the use of a single algorithm is possible by converting between distance and similarity measures (for example 
with heat kernel functions [2]), we elected to avoid this and apply manifold learning techniques specifically developed for each type of measure. This also means that additional parameter choices during conversion are avoided. We distinguish shape embedding coordinates $y_{i}^{s}$ from the appearance embedding $y_{i}^{a}$. In each case a target dimension, $d^{\alpha}, \alpha \in\{a, s\}$, needs to be chosen and the coordinates may be explicitly written as $y_{i}^{\alpha}=\left(y_{i, 1}^{\alpha}, \ldots, y_{i, d^{\alpha}}^{\alpha}\right)$.

The embedding coordinates $y_{i}^{s}$ and $y_{i}^{a}$ were then fused by uniformly scaling each set so that the variance of the first coordinate, $y_{i, 1}^{\alpha}, \alpha \in\{a, s\}$, becomes one and subsequently concatenating. This provides $\left(d^{a}+d^{s}\right)$-dimensional coordinates $\left(f_{a} y_{i}^{a}, f_{s} y_{i}^{s}\right)$ for the appropriate scale factors $f_{a}$ and $f_{s}$. Overlap in the descriptions of the data provided by the two input embeddings was then removed by applying a further dimensionality reduction step to the concatenated coordinates. This was achieved by finding all pairwise $L_{2}$ distances for the concatenated coordinates. A final Isomap step applied to the distances provides a combined coordinate embedding $y_{i}^{c}$ with dimension $d^{c}$.

\section{$3 \quad$ Data and Results}

\subsection{Image Data and Pre-processing}

The images studied were $140 \mathrm{~T} 2 \mathrm{~W}$ scans of neonatal subjects. Fast-spin echo scans were acquired on a 3 Tesla Philips Intera scanner $(\mathrm{TR}=1712 \mathrm{~ms}$, TE $=$ $160 \mathrm{~ms}, \mathrm{FA}=90^{\circ}$ ) with resolution $0.86 \times 0.86 \times 1 \mathrm{~mm}$. Mean gestational age (GA) at birth was $29.4 \pm 2.9$ weeks (range $23.4-34.9$ ) and mean age at scan was $36.8 \pm 4.8$ weeks (range 28.6-47.7). A clinical expert selected a reference scan displaying an intermediate level of maturation for the group (GA at birth/scan 32.0/36.3 weeks). All scans were aligned to the reference using affine transformations and resliced to remove global size and orientation differences. Each image pair was non-rigidly registered using free-form deformations [11] (FFDs). The resolution of the transformations was varied using a coarse-to-fine optimisation giving three FFDs, with control point spacings of 20, 10 and $5 \mathrm{~mm}$, for each pair of images.

\subsection{Results}

The target dimension, $d^{\alpha}, \alpha \in\{a, s, c\}$, for each embedding was empirically selected by varying it and calculating the correlation between the input edge

\begin{tabular}{|l||l|l|l||l|l|l|}
\hline & $y_{i, 1}^{c}$ & $y_{i, 1}^{s}$ & $y_{i, 1}^{a}$ & $y_{i, 2}^{c}$ & $y_{i, 2}^{s}$ & $y_{i, 2}^{a}$ \\
\hline \hline GA (S) & $0.93^{*}$ & $0.88^{*}$ & $0.92^{*}$ & 0.01 & 0.07 & 0.03 \\
W (S) & $0.91^{*}$ & $0.85^{*}$ & $0.89^{*}$ & 0.01 & 0.08 & 0.04 \\
HC (S) & $0.92^{*}$ & $0.88^{*}$ & $0.88^{*}$ & 0.10 & 0.18 & 0.07 \\
\hline GA (B) & 0.18 & 0.21 & 0.07 & $0.68^{*}$ & $0.61^{*}$ & $0.49^{*}$ \\
W (B) & 0.24 & 0.24 & 0.17 & $0.48^{*}$ & $0.45^{*}$ & $0.35 \dagger$ \\
HC (B) & 0.24 & 0.26 & 0.16 & $0.54^{*}$ & $0.56^{*}$ & $0.35 \dagger$ \\
\hline
\end{tabular}

Table 1. Correlation of clinical data and embedding components for each method. Gestational age (GA), weight (W) and head circumference $(\mathrm{HC})$ data were obtained at scan (S) or at birth (B). [* $\left.p<10^{-4}, \dagger p<10^{-3}\right]$ 

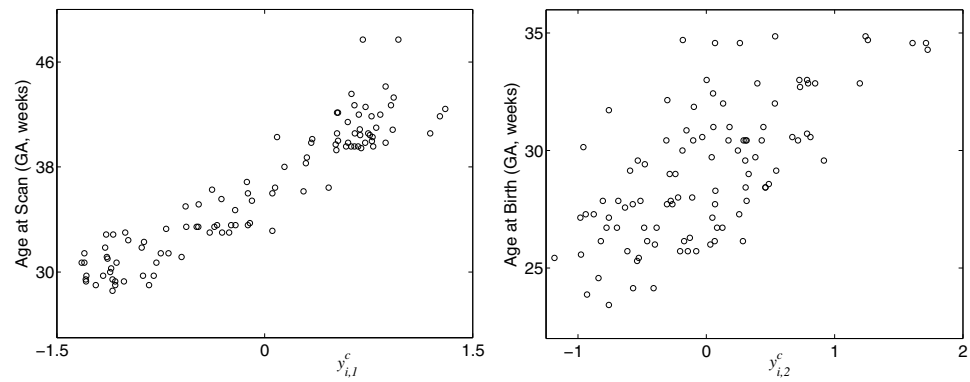

Fig. 2. Scatter graphs of combined embedding components with different age data. Left: $y_{i, 1}^{c}$ against gestational age (GA) at scan. Right: $y_{i, 2}^{c}$ against GA at birth.

weights and the embedding distances. For the Laplacian eigenmap method, the best correlation was obtained for $d^{a}=5$ and reduced thereafter. Using Isomap, the correlation increases asymptotically as the dimension increases and the minimal dimension for which the correlation exceeded 0.7 was selected to avoid over-fitting. This led to choices of $d^{s}=4$ and $d^{c}=5$. For the Isomap steps, the residual variance was 0.30 for $y_{i}^{s}$ and a much lower value of 0.05 when deriving $y_{i}^{c}$, reflecting already near-linear structure of the input data. The best control point spacing for the transformations was empirically chosen as above and matched expectations regarding the transformation resolutions for each type of measure with an intermediate spacing of $10 \mathrm{~mm}$ used to derive $y_{i}^{a}$ and a finer spacing of $5 \mathrm{~mm}$ for $y_{i}^{s}$.

The explanatory power of each set of embedding coordinates was assessed by its correlations with clinical data for the group which were recorded at birth and at scan. These were gestational age, head circumference and weight. The first component in each embedding, $y_{i, 1}^{s}$ and $y_{i, 1}^{a}$, correlates strongly with the measures obtained at scan (see Table1) and there are weaker but still significant correlations for the second components $y_{i, 2}^{s}$ and $y_{i, 2}^{a}$ with data obtained at birth.

Viewing the entries in Table 1 as a $2 \times 2$ block matrix with $3 \times 3$ elements, we note that the off-diagonal values are much lower than those on the diagonal which suggests orthogonality among the correlations. Within each diagonal block, the correlations for the combined embedding are, with one exception, greater than the corresponding correlations for the separate embeddings demonstrating the benefit of combining them.

Scatter graphs for the combined embedding components against ages at scan and at birth are shown in Figure 2. Visualisations are given in Figure 3 for the manifold structure of the image data provided by the first two components of the combined embedding. A continuous trajectory $y^{c}(t)$ through the combined embedding coordinates, parametrised by the age at scan $t$, was defined using kernel regression as $y^{c}(t)=\frac{1}{\nu} \sum_{i} K\left(t_{i}-t\right) y_{i}^{c}$ where $t_{i}$ is the age at scan for image $\mathcal{I}_{i}, K$ represents a Gaussian kernel and $\nu$ is a normalising constant. The trajectory (projected onto two dimensions) for our data is shown in Figure 3 as a solid line. Figure 4 illustrates reconstructions of images at five equally spaced 

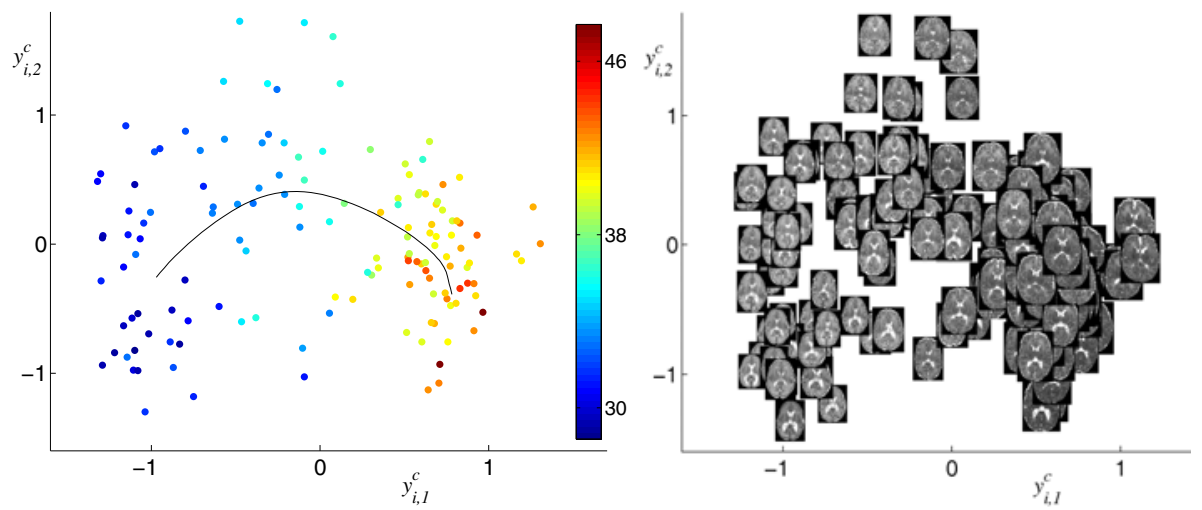

Fig. 3. Left: Scatter graph of combined embedding components $y_{i, 1}^{c}$ and $y_{i, 2}^{c}$ with colour coding for age at scan. The solid line shows a regressed trajectory for age at scan (see text). Right: The same scatter graph illustrated with image data.

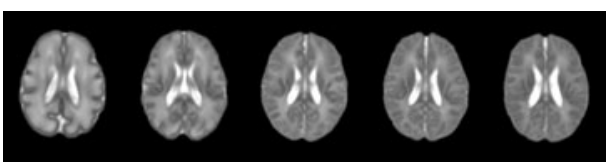

Fig. 4. Reconstructions of images along the trajectory for age at scan in Figure 3 (N.B. Original image data were normalised for global size).

points on the trajectory estimated using the method described in [5]. It should be noted that the reconstructions retain the global size normalisation of the pre-processed image data.

\section{Discussion}

We have presented a framework for applying manifold learning steps to imaging data using different measures of morphology. We have utilised shape and appearance measures although other applications may require different measures specific to their clinical context. Shape and appearance are important aspects of neonatal brain development and we derive their measures from image similarities and deformations. The measures provide coordinate embeddings after applying different manifold learning techniques appropriate to each type of measure used. Correlations with clinical data suggest overlap in the descriptions that the separate embeddings provide despite their separate derivations. This level of consistency across embeddings and the strong correlations with clinical data indicate that the manifold learning steps provide biologically relevant representations. The proposed method also combines separately obtained embeddings through scaling, concatenation and dimensionality reduction. While the separate embeddings may overlap in the information they provide, the improved correlations after combination also indicates some independence in the descriptions.

Considering the patterns of correlation between embeddings and clinical data, strong correlations between the first feature of all embeddings and age at scan is 
perhaps unsurprising as it is the dominant factor affecting brain shape and MR appearance. It is also expected that correlations with weight and head circumference should parallel correlations with age (because size measures are themselves correlated with age) but this at least provides further proof of principle for the results. The second components, however, correlate with gestational age at birth which suggests that, after scan age, variation in brain morphology is mainly determined by age at birth. A possible explanation for this is that gestational age at birth measures the degree of prematurity for an infant which in turn may affect morphology. The apparent orthogonality among the observed correlations (Table 1) suggests an independence to the morphological features described by the first and second components in each embedding.

Finally we have shown in a preliminary example how trajectories may be described in the embedding coordinates using kernel regression and, following the work in [5], how image reconstructions of such a trajectory may be obtained. The trajectory parameter was age at scan but clearly other clinical covariates can be used. This ability to use the proposed approach to characterize change in cohorts, with respect to chosen covariates, has clear clinical potential, for example in identifying pathology or in tracking growth.

\section{References}

1. Langs, G., Paragios, N.: Modeling the structure of multivariate manifolds: Shape maps. In: Proc. CVPR 2008. IEEE, Los Alamitos (2008)

2. Belkin, M., Niyogi, P.: Laplacian eigenmaps for dimensionality reduction and data representation. Neural Computation 15(6), 1373-1396 (2003)

3. Aljabar, P., Rueckert, D., Crum, W.: Automated morphological analysis of magnetic resonance brain imaging using spectral analysis. NeuroImage 43(2), 225-235 (2008)

4. Tenenbaum, J., de Silva, V., Langford, J.: A global geometric framework for nonlinear dimensionality reduction. Science 290(5500), 2319-2323 (2000)

5. Gerber, S., Tasdizen, T., Joshi, S., Whitaker, R.: On the manifold structure of the space of brain images. In: Yang, G.-Z., Hawkes, D., Rueckert, D., Noble, A., Taylor, C. (eds.) MICCAI 2009. LNCS, vol. 5761, pp. 305-312. Springer, Heidelberg (2009)

6. Hamm, J., Davatzikos, C., Verma, R.: Efficient large deformation registration via geodesics on a learned manifold of images. In: Yang, G.-Z., Hawkes, D., Rueckert, D., Noble, A., Taylor, C. (eds.) MICCAI 2009. LNCS, vol. 5761, pp. 680-687. Springer, Heidelberg (2009)

7. Wolz, R., Aljabar, P., Hajnal, J., Hammers, A., Rueckert, D.: The Alzheimer's Disease Neuroimaging Initiative: LEAP: Learning embeddings for atlas propagation. NeuroImage 49(2), 1316-1325 (2010)

8. Beg, M., Miller, M., Trouvé, A., Younes, L.: Computing large deformation metric mappings via geodesic flows of diffeomorphisms. IJCV 61(2), 139-157 (2005)

9. Ashburner, J., Friston, K.: Voxel-based morphometry - the methods. NeuroImage 11(6), 805-821 (2000)

10. Gaser, C., Nenadoc, I., Buchsbaum, B., Hazlett, E., Buchsbaum, M.: Deformationbased morphometry and its relation to conventional volumetry of brain lateral ventricles in MRI. NeuroImage 13, 1140-1145 (2001)

11. Rueckert, D., Sonoda, L., Hayes, C., Hill, D., Leach, M., Hawkes, D.: Non-rigid registration using free-form deformations: Application to breast MR images. IEEE Trans. Medical Imaging 18(8), 712-721 (1999) 